Post-hoc-Analyse kam sogar zu dem Schluss, dass Digoxin die Mortalität bei Vorhofflimmern-Patienten mit schwer reduzierter Ejektionsfraktion mindert.

\section{Das Tralala der Studieninterpreten}

Letztlich kranken viele einschlägige Studien an Verzerrungen (einschließlich des Verschreibungsbias). Nur prospektive, randomisierte Studien lieferten sichere Daten, betonte Bauersachs und zeigten, wie das Digitalis-Paradox gelöst werden könne. Die seit August 2015 laufende DIGIT-HF Studie („DIGitoxin to Improve ouTcomes in patients with advanced chronic systolish Heart Failure") mit 2.200 Teilnehmern soll definitiv für Klarheit sorgen, ob Digitoxin bei schwerer Herzschwäche das Leben verlängern und die Krankenhausbehandlung reduzieren kann. Bis dahin gelten laut Bauersachs folgende Orientierungspunkte für den Einsatz von Digitalis bei Patienten mit systolischer Herzinsuffizienz:

-Allem voran ist eine kritische Indikationsstellung sowie individualisierte Therapieeinstellung und -kontrolle nötig, was übrigens für alle Herzinsuffizienzmedikamente gilt;

-Insuffizienzpatienten mit einer Auswurffraktion von $<35 \%$;

_ Im Stadium III und IV trotz Standardtherapie mit Betablocker, ACE-Hemmer, MRA und evtl. Ivabradin;

-Hospitalisierungen wegen Dekompensation;

- Tachyarrhythmie/Ruhefrequenz $>80$ 90/min trotz Betablocker;

_Eher niedrige Dosis, keine LoadingDosis, außer bei Vorliegen einer Tachyarrhythmia absoluta;

_Spiegelkontrollen vor allem bei Digoxin; - Bei Niereninsuffizienz und älteren Patienten Digitoxin statt Digoxin.

\section{ARNI - eine neue Hoffnung}

Vom Oldtimer Digitalis ging es ansatzlos zum neuesten Kandidaten auf der Liste pharmakotherapeutischer Innovationen: dem ersten Vertreter der ARNIFamilie (Angiotensin-Rezeptor-Neprilysin-Inhibitor), in den USA und Europa zugelassen als Entresto ${ }^{\circ}$. Prof. Sven Waßmann, Isar Herzzentrum München, hält

\section{pci kPngress 2015}

die neue Kombination für einen Quantensprung, jedoch enthielt sein Vortragstitel noch ein Fragezeichen. Die Argumente für die freudige Botschaft lieferte die vielzitierte PARADIGM HFStudie, in der sich der ARNI dem ACE-Hemmer Enalapril hinsichtlich Todesfälle und Hospitalisierungrate signifikant überlegen zeigte. Waßmann verwies darauf, dass heute noch die Mortalität fünf Jahre nach Diagnosestellung $50 \%$ beträgt - Zahlen, die schon vor über einem Jahrzehnt genannt wurden. Offenbar sind die Statistiken so grob, dass sie nur große Fortschritte abbilden oder aber die positiven Ergebnisse in den letzten zehn Jahren waren nur für die einschlägigen Studien relevant, nicht aber für die Breitenversorgung.

Die Pharmahoffnungen setzen auf die ARNI-Familie. Ob damit ein klinisch relevanter Fortschritt, ein Durchbruch oder Quantensprung erzielt wird, müssen die nächsten Jahre zeigen, sobald auch alle Langzeit-Sicherheitsaspekte zur Zufriedenheit ausfallen.

Dr. med. Jochen Aumiller

\title{
Kathetervirtuosen im Münchner Haus der Kunst
}

Sieben Mal war die BMW-Welt Präsentationsort des P.C.I.-Kongresses, dann scheint es bei den Organisatoren mächtig gekracht zu haben. Denn zum achten P.C.I. lud Gründungsvater Prof. Sigmund Silber ins Münchner Haus der Kunst ein,

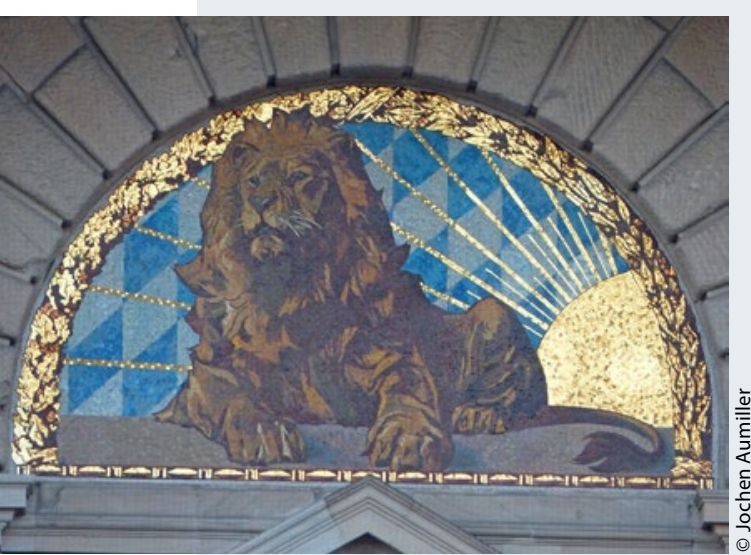

Diesmal wurde der P.C.I-Kongress im Münchner Haus der Kunst ausgetragen. der abgespaltene pci-Heartlive lockte nach Stuttgart in die Carl Benz Arena schon Ende Oktober 2015. Und die BMWWelt? War wieder exklusiv dem Automobilhandel vorbehalten.

Fakt ist, dass sich mit dieser überraschenden "Zellteilung" das Fort- und Weiterbildungsangebot in Sachen interventionelle Kardiologie kräftig erweitert hat.

Aber warum ins Haus der Kunst? Man wundert ich jedes Mal, wenn man vor Hitlers Münchner Musentempel steht, der 1945 von den Bomben der Alliierten verschont wurde, anders als die nahegelegene Altstadt, von der überwiegend Schutt und Asche übrig blieb. Des Rätsels Lösung: Rechtzeitig wurden Tarnnetze über den Gebäudekomplex gezogen, sodass er von oben wie ein Teil des angrenzenden Englischen Gartens verkannt wurde.

Von solchen Camouflage-Künsten war auf dem P.C.I. nicht die Rede, wie man überhaupt historische Bezüge überging. Im Eröffnungsvortrag stand „Das gebro- chene Herz" vornehmlich aus feuilletonistischer Sicht im Mittelpunkt. Immerhin erfuhr man, dass heiraten und vor allem verheiratet bleiben als herzgesund einzustufen ist; im Gegensatz zu den geschiedenen Männern, deren Herz früher bricht, übrigens unabhängig davon, ob die Hautfarbe weiß oder schwarz war. In der US-Statistik aus den Jahren 1959 bis 1961 waren die Frauen leider ausgeklammert.

Das Programm des zweitägigen Kongresses umarmt gewissermaßen das ganze Gebiet der interventionellen Kardiologie (Elektrophysiologie, koronare und Klappeneingriffe) und sah auch Workshops vor, in denen Tipps und Tricks vermittelt wurden entweder zum radialen Zugang oder schwerpunktmäßig auch für die TAVI, für die sich die Kardiotechnik diverse Systeme hat einfallen lassen.

Wir haben für die Berichterstattung das Kapitel Herzinsuffizienz gewählt, eine zentrale Herausforderung für alle Beteiligen: Kardiologen und Herzchirurgen. 